\title{
„Die Test-and-treat-Strategie kann nicht empfohlen werden!"
}

\author{
In der neuen Leitlinie zu Helicobacter pylori haben sich die Experten gegen die Strategie des \\ Infektionsnachweises mit nachfolgender Eradikation ohne Endoskopie bei Dyspepsie ausge- \\ sprochen. Wen soll man nun auf den Magenkeim testen? Wir fragten nach bei Prof. Fischbach, \\ der als DGVS*-Mitglied federführend an der Leitlinie mitgearbeitet hat.
}

\section{$?$}

Prof. Dr. med. Wolfgang Fischbach Klinik II des Klinikums Aschaffenburg
Chefarzt der Medizinischen

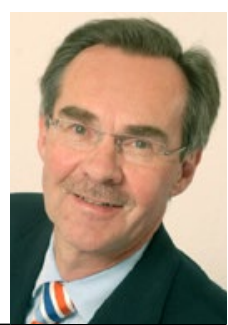

MMW: Herr Professor Fischbach, wen soll man in der allgemeinärztlichen Praxis auf $\mathrm{H}$. pylori testen?

Fischbach: Vor allem Patienten, die aufgrund von kardiovaskulären Problemen eine ASS-Dauermedikation erhalten sollen oder die wegen einer Schmerzproblematik für eine längerfristige Therapie mit NSAR anstehen, und zwar jeweils eingegrenzt auf Patienten mit einer Ulkusanamnese. Hierfür gibt es in der Leitlinie eine starke Soll-Empfehlung. Das Problem ist, dass in Deutschland genau für diese Patienten nicht invasive Nachweisverfahren wie Stuhltest oder Atemtest nicht zugelassen sind; hieran müssen wir mit den Entscheidungsträgern arbeiten.

MMW: Laut Leitlinie soll die Auswahl des Tests nach der Indikation erfolgen. Was bedeutet das für die Praxis?

Fischbach: Hier geht es vor allem um die Abgrenzung des serologischen Antikörpernachweises: Dieser hat für die Abklärung einer aktiven H.-pylori-Infektion keinen Stellenwert. Ein positiver Antikörpertest zeigt ja nur an, dass irgendwann eine Infektion stattgefunden hat. Bei der Fragestellung, ob eine akute Infektion vorliegt, haben Sie zwei Möglichkeiten: Gibt es keinen Grund für eine
Endoskopie, können Sie einen nicht invasiven Test - entweder den ${ }^{13} \mathrm{C}$-Atemtest oder den Stuhl-Antigennachweis durchführen. Wenn man sich aber von einer Endoskopie verspricht, dass sie Informationen zum Beschwerdebild und dahinterstehenden Ursachen liefert, wird man den Helicobacter-Nachweis im Rahmen der Endoskopie führen. Das ist am besten mittels Urease-Schnelltest oder durch die Histologie möglich.

MMW: Was ist der Hintergrund für die „Kann"Empfehlung für eine Eradikation bei funktioneller Dyspepsie?

Fischbach: Es gibt Daten, dass etwa $8 \%$ bis $10 \%$ der Patienten mit funktioneller Dyspepsie längerfristig von einer Eradikation profitieren, nicht weniger und nicht mehr. Für diese Patienten gibt es wenig erfolgversprechende Alternativen; wir können es hier mit der Eradikation versuchen. Was wir nicht empfehlen, ist die Test-and-treat-Strategie! In Deutschland haben wir im Vergleich zu Südeuropa eine relativ geringe Durchseuchungsrate. Wir müssten daher sehr viele Patienten untersuchen, um einzelne mit Helicobacter herauszufischen. Das zweite Argument: Die Endoskopie zur Abklärung dyspeptischer Beschwerden ist hierzulande problemlos und relativ kostengünstig verfügbar. Wir empfehlen daher zur Abklärung einer Dyspepsie die einmalige Endoskopie, durchaus auch mit HelicobacterDiagnostik.
MMW: Wen soll man unter dem Gesichtspunkt der Prävention eines $\mathrm{Ma}$ genkarzinoms testen und behandeln? Fischbach: Vor allem die Patienten, bei denen in der Familie Fälle von Magenkrebs bekannt sind. Außerdem Patienten, die wir wegen eines gutartigen Adenoms oder Magenfrühkarzinoms endoskopisch oder mittels einer Magenteilresektion behandelt haben. Die dritte Risikogruppe sind Patienten mit einer Corpus-dominanten oder Pan-Gastritis. Die vierte Gruppe, und das ist neu in der Leitlinie, sind Patienten, die - z. B. wegen einer Refluxkrankheit - mehr als ein Jahr lang kontinuierlich mit einem PPI behandelt werden.

\section{MMW: Was sagt die} neue Leitlinie zur Über-

\section{prüfung des Therapieerfolgs?}

Fischbach: Im Gegensatz zur alten Fassung fordert die neue Leitlinie generell eine Kontrolle des Eradikationserfolgs, unabhängig von der zugrunde liegenden Indikation. Hierfür bieten sich besonders der Atemtest oder der Stuhltest an. Und für diese Indikation sind diese beiden Tests auch zugelassen! Zwischen Abschluss der Eradikationsbehandlung und Kontrolle sollte ein Zeitintervall von mindestens vier Wochen liegen. Eine säurehemmende Therapie mit einem PPI sollte nach Möglichkeit zwei Wochen zuvor ausgesetzt werden.

Interview: Dr. Elke Oberhofer

${ }^{*}$ Deutsche Gesellschaft für Verdauungs- und Stoffwechselkrankheiten 